Cite this: RSC Advances, 2013, 3, 10323

Received 20th November 2012, Accepted 2nd April 2013

DOI: $10.1039 / c 3 r a 22974 b$

www.rsc.org/advances

\title{
Synchrotron small angle X-ray scattering for the evaluation of the interaction of silica nanotubes with lipid membranes
}

\author{
Cláudia Nunes, $\uparrow^{\star a}$ Célia T. Sousa, ${ }^{\star b}$ Mariana P. Proença, ${ }^{b}$ A. Apolinário, ${ }^{b}$ José L. F. \\ C. Lima, ${ }^{a}$ Salette Reis, ${ }^{a}$ João P. Araújob and Marlene Lúcio ${ }^{a}$
}

\begin{abstract}
Synchrotron X-ray scattering measurements were used to assess the interaction of silica nanotubes (SNTs) with membrane model systems in conditions that mimic the physiological $\mathrm{pH}$ of healthy $(\mathrm{pH} 7.4)$ and pathological ( $\mathrm{pH}$ 5.0) cellular membranes. SNT arrays were synthesized combining the sol-gel method with a porous anodic alumina (PAA) template assisted approach. The inner surfaces of the SNTs were functionalized with aminopropylethoxysilane (APTES). Scanning electron microscopy (SEM) and transmission electron microscopy (TEM) were used to characterize the nanocarriers. A therapeutic antiinflammatory drug (naproxen) was loaded into the SNTs by electrostatic interactions between the negatively charged carboxyl group of the drug and the protonated inner surface of the nanotubes. Small angle X-ray scattering (SAXS) measurements indicate that the unloaded SNTs do not interact significantly with both membranes. The negligible membrane disordering effect demonstrated by the unloaded SNTs may correlate with a desirable lack of membrane toxicity of these nanocarriers. Furthermore, the biophysical effect on membrane structure presented a similar profile at pH 5.0 for the drug (nonencapsulated) and for SNTs loaded with the drug indicating that SNTs are able to release the drug content under pathological conditions.
\end{abstract}

\section{Introduction}

Silica nanoparticles have many advantages, since they are extremely stable and their size, shape and porosity can effortlessly be controlled. ${ }^{1}$ In addition, there is no enlargement or porosity change upon $\mathrm{pH}$ variation; no vulnerability to microbial attack and these particles have also been shown to effectively protect entrapped molecules (enzymes, drugs, etc.) against degradation. ${ }^{2}$ Silica-based particles are also known for their biocompatibility ${ }^{3,4}$ and their easy surface functionalization that allows the modification of the hydroxyl groups on the surface with amines, thiols, carboxyls and methacrylate, according to the desired application. ${ }^{5}$

Moreover, high aspect ratio inorganic nanoparticles have attracted great interest for their vast applications. ${ }^{6}$ In particular, nanotubes offer the possibility of differential functionalization that can provide inner voids for loading drug molecules or imaging agents and the outside surface for targeting moieties, antifouling agents or different kinds of

${ }^{a}$ REQUIMTE, Departamento de Química, Faculdade de Farmácia, Universidade do Porto, Portugal.E-mail: claununes@gmail.com

${ }^{b}$ IFIMUP and IN-Institute of Nanoscience and Nanotechnology and Dep. Física e Astronomia, Univ. Porto, Rua do Campo Alegre 687, 4169-007 Porto, Portugal. E-mail: celiasousa@fc.up.pt

$\dagger$ These authors have contributed equally. molecules. Furthermore, since the innovation of template assisted synthesis using porous anodic alumina (PAA), it is possible to obtain monodispersed nanotubes of nearly any material with the desired length and pore diameter just by changing the anodization conditions. ${ }^{7}$

Nowadays, effective disease targeting represents an enormous biomedical challenge in the treatment of inflammatory diseases and other serious illnesses, since the lack of selectivity of current therapeutic agents results in numerous severe deleterious side effects, including organ and tissue damage. ${ }^{8}$ This constitutes a key limitation of current therapies as those drugs must often be administered at suboptimal doses to reduce cytotoxicity. Therefore, it becomes increasingly important to develop drug delivery systems that can efficiently target based on subtle molecular alterations that distinguish specifically the inflamed or other diseased cells, from the normal ones. In this context, a controlled drug release strategy can be achieved based on the sensitivity of the nanocarrier system to the $\mathrm{pH}$ conditions. This strategy applied to the case of non-steroidal anti-inflammatory drugs (NSAIDs) assures that the drugs are only released at the inflamed tissues (that have $\mathrm{pH}$ 5.0) thus minimizing the side effects of these drugs to healthy tissues (that have a $\mathrm{pH}$ of 7.4). Additionally, before reaching their targets and during their body distribution, drug delivery systems must cross biological membranes. Therefore 
it is of utmost importance to evaluate the interaction of nanocarrier systems with membranes. The study of the interaction between nanoparticles and membranes gains deeper importance in the case of silica nanotubes (SNTs) since their use could be questioned by their capacity to insert into the lipid bilayers disrupting the biophysical integrity of the membranes. Consequently, SNTs containing NSAIDs should carry the drug to the inflammation site without considerable interaction with the cellular membranes of healthy tissue.

For the pointed reasons, the present study is focused on assessing the effect of SNTs on membrane structure and also the $\mathrm{pH}$ dependent release of the incorporated NSAID (naproxen) in the inflamed acidic tissue conditions. The SNTs were produced by a template assisted method using PAA. After synthesis, the voids of the SNTs were functionalized with an aminosilane, generating an inner polycationic surface, to which a negatively charged NSAID (naproxen) was electrostatically bonded. The PAA templates as well as the SNTs, before and after functionalization, were morphologically characterized using Scanning Electron Microscopy (SEM) and Transmission Electron Microscopy (TEM). The structural information about the interaction between the SNTs (loaded and unloaded) or the naproxen with the lipid bilayers was accessed by a technique widely used in biophysical studies: small angle X-ray scattering (SAXS). Moreover, this technique is also very useful for assessing the loading and the release of drugs. ${ }^{9}$ Synthetic lipid bilayers of 1,2-dipalmitoyl-sn-glycero-3phosphocholine (DPPC) at $\mathrm{pH} 7.4$ were used as biomimetic model systems, mimicking biological healthy membranes that possess a composition rich in phosphocholine. Moreover, DPPC is an endogenous component of the joints and represents approximately $45 \%$ of the total synovial fluid lipid component. ${ }^{10}$ Hence, lipid bilayers of DPPC at $\mathrm{pH} 5.0$ mimicked the biological inflammation targets of NSAIDs.

Overall the gathered results provided insight about the SNTs cellular uptake and corroborate the preferential effect of naproxen on the membranes in pathological conditions.

\section{Experimental}

For the growth of SNTs we used PAA templates obtained by a standard two-step anodization method of high-purity (99.997\%) Al foils. ${ }^{11}$ After an electropolishing pre-treatment, the $\mathrm{Al}$ foils were anodized in a $0.3 \mathrm{M}$ oxalic acid solution at 4 ${ }^{\circ} \mathrm{C}$ and under an applied potential of $40 \mathrm{~V} \cdot{ }^{12}$ The first anodization was carried out for $24 \mathrm{~h}$ while the second lasted $15 \mathrm{~min}$. These anodization conditions resulted in nanopores with an average diameter of $40 \mathrm{~nm}$ and $500 \mathrm{~nm}$ in length. The polymeric SNTs were prepared by combining the PAA template assisted method with a sol obtained under acidic conditions using tetraethyl orthosilicate (TEOS) as a silica precursor and their inner surface was also functionalized with aminopropylethoxysilane (APTES) following a procedure described in a previous work. ${ }^{13}$
After the inner surface functionalization with aminosilane, the PAA was dissolved and the SNTs that were inside of the PAA template were resuspended in a solution of $\mathrm{KOH}(0.1 \mathrm{M})$ for $10 \mathrm{~min}$, centrifuged at $35000 \mathrm{G}$ for $25 \mathrm{~min}$ and washed with bideionised water. Then the SNTs were again resuspended in a Hepes buffered solution $(\mathrm{pH}=7.4)$ of naproxen $(10 \mu \mathrm{M})$ and incubated in the dark for $2 \mathrm{~h}$ with continuous agitation. In order to wash and remove the excess naproxen that was not attached to the inner surface of the SNTs, the suspension was again washed with bideionised water and centrifuged three times.

The size and morphology of the nanostructures were determined by Scanning Electron Microscopy (SEM) analysis in a FEI Quanta 400 FEG SEM and by Transmission Electron Microscopy (TEM) in a LEO 906E Leica.

SAXS experiments were performed at the beam line A2 of Doris III at HASYLAB (DESY, Hamburg, Germany) with a monochromatic radiation of wavelength $0.15 \mathrm{~nm}$. The SAXS detector was calibrated with rat-tail tendon. Heating scans were performed at a rate of $1{ }^{\circ} \mathrm{C} \mathrm{min}{ }^{-1}$ in the range $10{ }^{\circ} \mathrm{C}$ to 52 ${ }^{\circ} \mathrm{C}$. Data was recorded for $10 \mathrm{~s}$ every minute. Static exposures were also taken below and above the main transition temperature and compared with the same temperature in the heating cycles to check for possible radiation damage. In order to minimize the X-ray exposure to the sample, a shutter mounted before the sample was kept closed when no data was acquired. Lipid dispersions composed of multilamellar vesicles (MLVs) of DPPC mixed with naproxen, SNTs and SNTs loaded with naproxen were prepared at $\mathrm{pH} 5.0$ and $\mathrm{pH} 7.4$ according to a well described method. ${ }^{14,15}$ The SNT lipid dispersions were prepared with a fixed concentration of $1 \times$ $10^{11}$ SNTs per mL; the naproxen loaded SNTs had $162 \mu \mathrm{g}$ of incorporated naproxen ${ }^{13}$ and the naproxen lipid dispersions contained $30 \mu \mathrm{g} \mathrm{mL}^{-1}$ of drug.

\section{Results and discussion}

\section{SNT characterization}

The SNTs were easily grown inside the pores of PAA templates by a combination of anodization and sol-gel techniques. Furthermore, the SNTs production method presented herein allows an accurate tailoring of the SNTs dimensions, the size homogeneity and distinct functionalization of both inner and outer walls.

Detailed imaging studies were made on the SNTs in the PAA template and after the template was removed. The studies include TEM and FE-SEM. Fig. 1 (a) shows a typical FE-SEM image of the PAA surface. Self-ordered arrays of uniform pores are observed with a pore diameter of $40 \mathrm{~nm}$ and an inter-pore distance of $105 \mathrm{~nm}$. In Fig. 1 (b) a representative SEM image of a sample of the SNTs is shown, in which the tubular nanostructure is clearly visible. This indicates that a onedimensional silica material has been prepared in the form of nanotubes. The SNTs have an outer diameter of $40 \mathrm{~nm}$, replicating the pore size of the PAA that was used. This indicates that the size, shape and organized hexagonal 

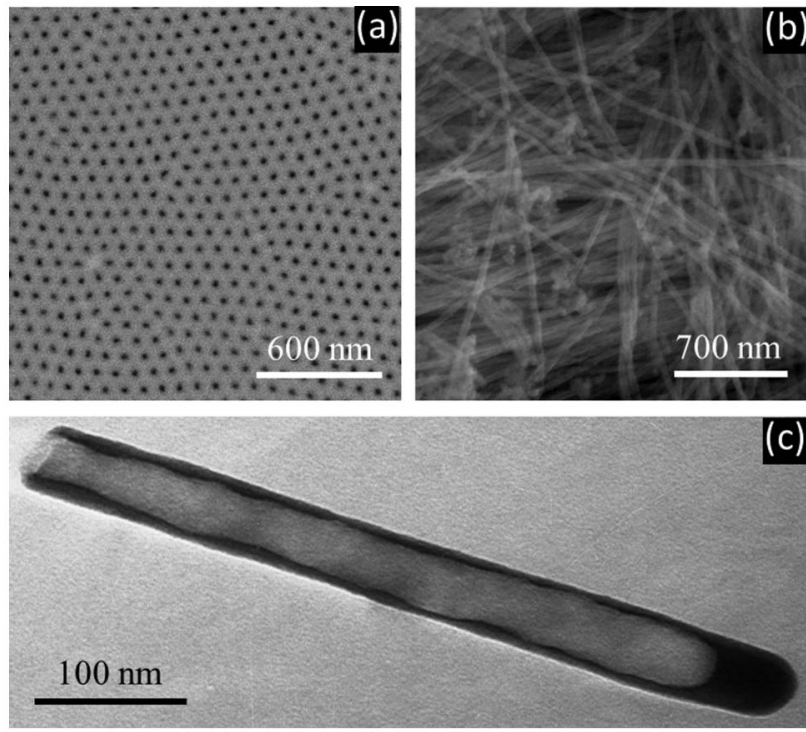

Fig. 1 (a) Surface FE-SEM image of a PAA template; FE-SEM image of the SNTS after complete removal of the PAA template and (c) TEM image of an isolated SNT.

packing of the NTs are controlled by the characteristics of the template. Further structural characterization of the SNTs was performed by TEM (Fig. 1 (c)). The TEM image confirms that the outer diameter of the SNT $(40 \mathrm{~nm})$, corresponds to the pore size of the PAA template used. The image also shows an inner diameter of about $35 \mathrm{~nm}$ and a wall thickness of $5 \mathrm{~nm}$. Furthermore it is possible to see that only one of the ends of the tube is open.

\section{SAXS measurements}

DPPC molecules spontaneously form lyotropic lamellar phases in excess water, whose structure and long-range organization are temperature dependent. ${ }^{15,16}$ As temperature is increased from room temperature, DPPC presents three different phases: gel phase $\left(\mathrm{L}_{\beta},\right)$, ripple phase $\left(\mathrm{P}_{\beta},\right)$ and liquid-crystalline phase $\left(\mathrm{L}_{\alpha}\right) . \mathrm{L}_{\beta^{\prime}}$ and $\mathrm{P}_{\beta^{\prime}}$ are ordered lamellar phases with tilted acyl chains predominantly in all trans conformation, and $\mathrm{L}_{\alpha}$ is the lamellar fluid phase with disordered acyl chains due to conformational changes (trans-gauche isomerization). ${ }^{17}$ In this work, the degree of perturbation of the $L_{\beta}$, and the $L_{\alpha}$ phases of DPPC (the most biologically relevant) by SNTs loaded with naproxen was evaluated by X-ray diffraction patterns at small angles (SAXS), yielding information on the long range bilayer organization. In order to assess the effective release of the drug in a $\mathrm{pH}$ dependent manner the studies were carried out with SNTs loaded with naproxen at $\mathrm{pH} 7.4$ and 5.0 (the $\mathrm{pH}$ of inflamed cells). Studies of the interaction of both naproxen and the unloaded SNTs with DPPC were also performed, making clear the contribution of the SNTs in the interaction of naproxen with the membranes.

Lamellar lattice constants, $d$, were calculated from the small angle Bragg reflections using the equation: $s=n / d$; where $s$ is the scattering vector and $n$ is the order of the reflection $(n=1$, $2, \ldots)$. To obtain a more precise position for $s$, the diffraction
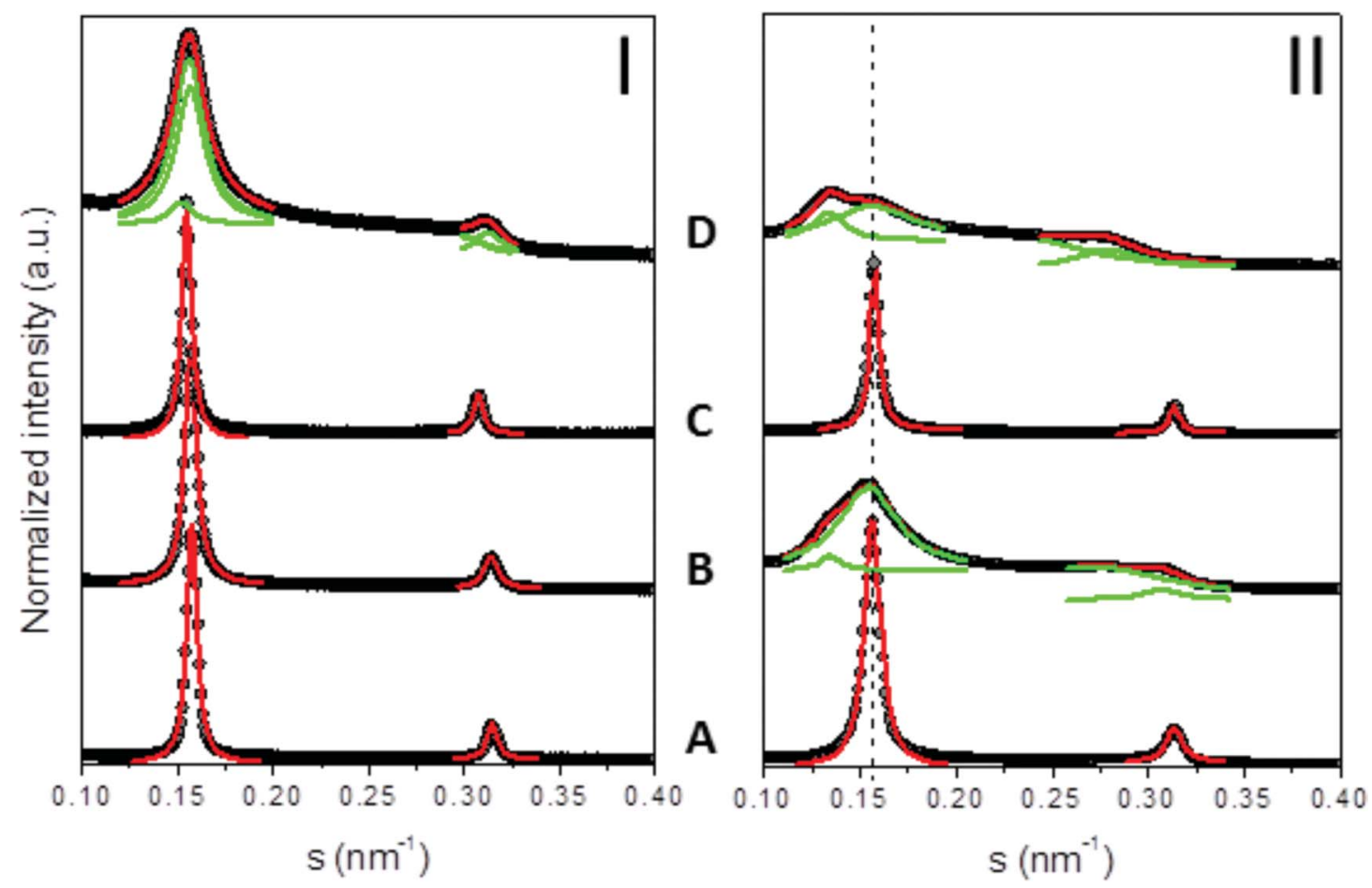

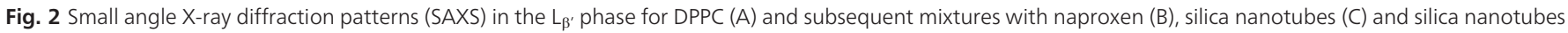

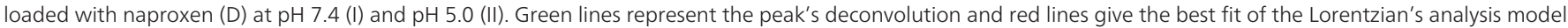
to the scattered intensities. 
Table 1 Long distance $(d)$ and correlation length $(\xi)$ determined from SAXS diffraction patterns, at $20{ }^{\circ} \mathrm{C}$ and $50{ }^{\circ} \mathrm{C}$ and at pH 5.0 and 7.4 , respectively

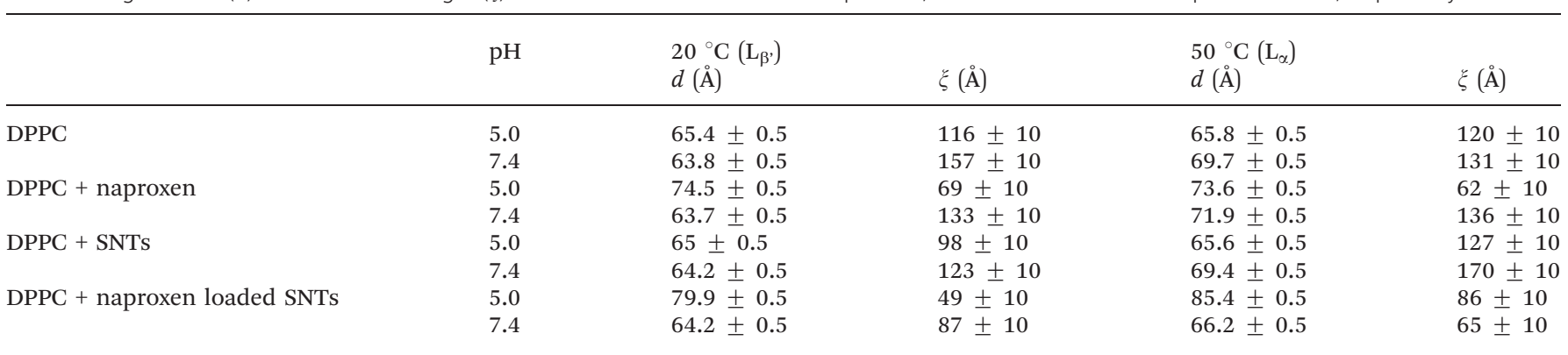

peaks were fitted with Lorentzians and the positions of maximum intensities and full widths of peaks at one half of their intensity were determined.

The SAXS analysis of the effects of naproxen, SNTs and SNTs loaded with naproxen, towards the DPPC bilayer at $\mathrm{pH} 7.4$ (Fig. 2-I) shows that no significant differences are observed in the $L_{\beta}$, phase of DPPC. The position of the Bragg peaks was not significantly affected by the presence of either the drug or the nanosystem and the $d$ values remain about the same as the one for the pure DPPC (Table 1). However, the Bragg peaks corresponding to mixtures of the lipid and the SNTs loaded with naproxen show a higher full width at half maximum than the diffraction peak corresponding to pure DPPC (Fig. 2-I), indicating that the correlation between the bilayers $(\xi)$ is slightly decreased by the presence of the nanosystem, which points to a decrease in the lipid order. In fact, three peaks are deconvoluted and correspond to the contributions of the pure lipid, the lipid:naproxen, and the lipid:SNTs. At pH 5.0 and still in the $\mathrm{L}_{\beta}$, it is observed that naproxen alone leads to the splitting of the first-order Bragg peak (Fig. 2B-II). One peak is located at a $d$ value of $6.41 \mathrm{~nm}$ (same as the pure DPPC), and the other reflection has a spacing of $7.45 \mathrm{~nm}$. The coexistence of a non-influenced lipid phase and an influenced lipid phase indicates that at the tested concentration there are not enough drug molecules to reach a homogeneous distribution of the drug within the lipid membrane. The same kind of behavior can be observed for the SNTs loaded with naproxen, with the exception that in this case (Fig. 2D-II) the influenced phase has a higher contribution and this can only be attributed to the fact that at this $\mathrm{pH}$ the electrostatic bond between the drug
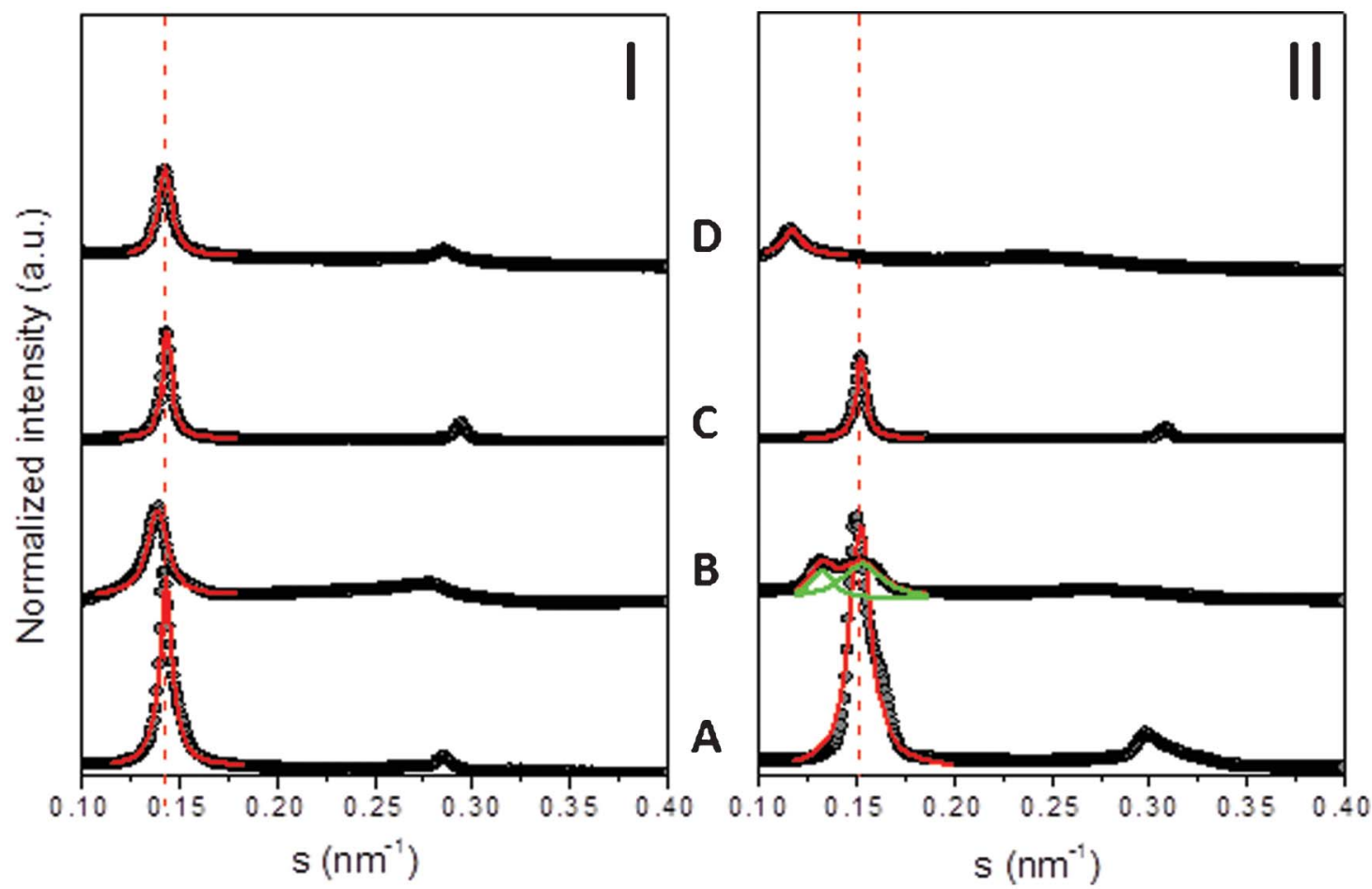

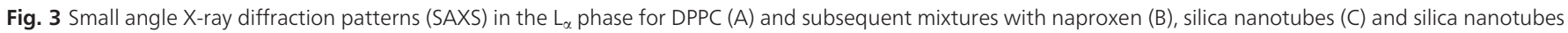

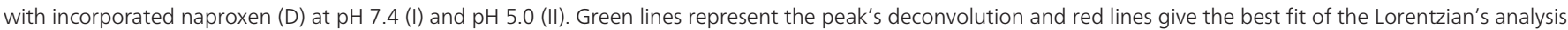
model to the scattered intensities. 
and the SNTs is weakened causing a release of naproxen from the SNTs. Indeed, the SNTs alone do not provoke any perturbing effect (Fig. 2C-II) in the lipid structure, confirming that the effects observed for the SNTs loaded with naproxen (Fig. 2D-II) must be due to the drug release from the nanotubes.

Looking to the $\mathrm{L}_{\alpha}$ phase (Fig. 3), the addition of naproxen at pH 7.4 (Fig. 3B-I) led to a slight increase in the $d$ value to 7.15 nm (Table 1). Such an increase in the long spacing could be the result of a change in the hydration behavior of DPPC due to the interaction with naproxen in the disordered liquidcrystalline phase. Once more, the SNTs alone have no relevant effect on the structure of the membrane, and the same is observed for the SNTs loaded with naproxen, which means that no naproxen was released from the interior of the nanotubes.

At $\mathrm{pH}$ 5.0, in the $\mathrm{L}_{\alpha}$ phase it is possible to see that naproxen leads again to the splitting of the Bragg peaks (Fig. 3B-II), which is a sign of the coexistence of a non-influenced phase and an influenced one. On the other hand, the mixture of lipid
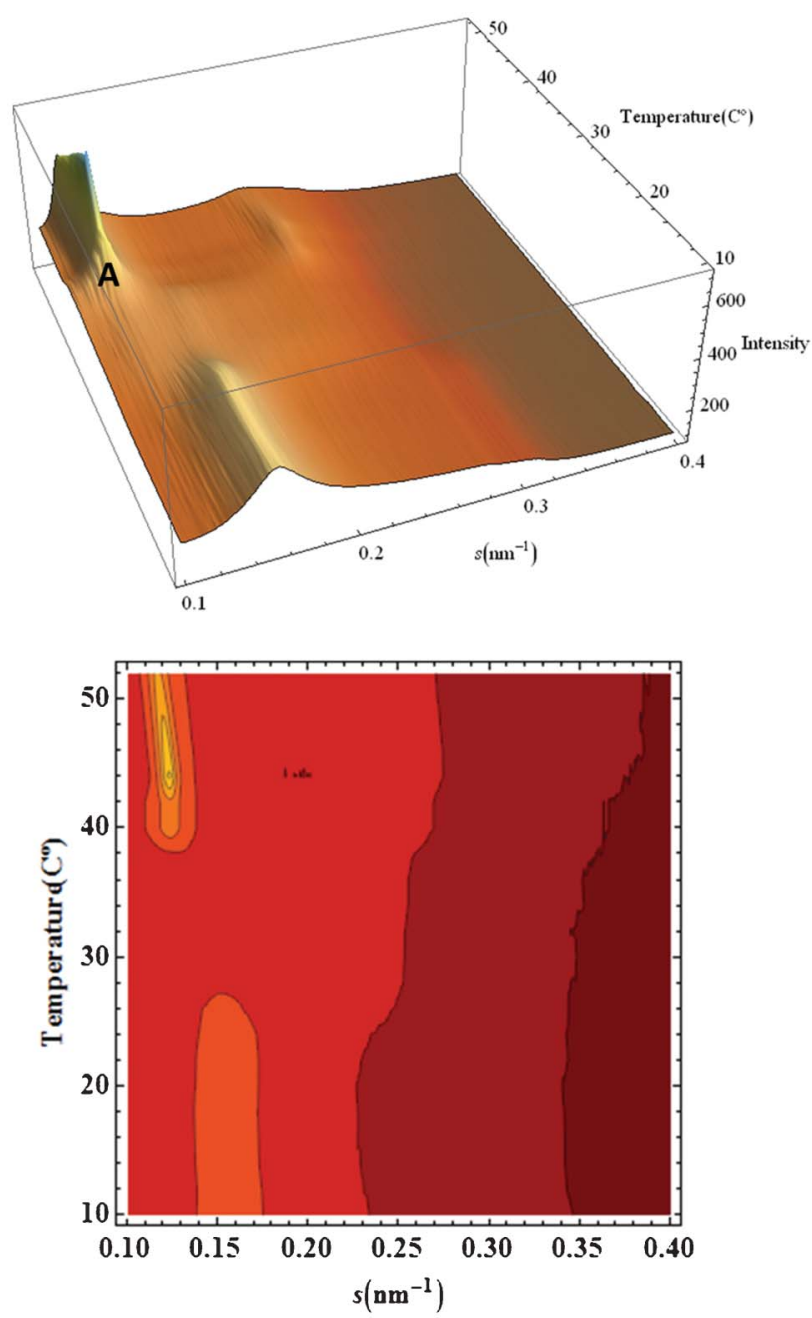

Fig. 4 Three-dimensional plot of a surface representing small angle X-ray diffraction (SAXS) heating scan from $10-52{ }^{\circ} \mathrm{C}$ for the DPPC mixture with SNTs loaded with naproxen at $\mathrm{pH}$ 5.0. with SNTs loaded with naproxen presents only one Bragg peak with a $d$ value of $8.62 \mathrm{~nm}$ (Fig. 3D-II). This increase can only be due to the release of naproxen and to the interaction of the drug with the lipid bilayer, as the SNTs alone produced no visible effect (Fig. 3C-II). The interaction observed (increase in $d$ values) results in the change in the hydration behavior of DPPC, enhancing the water layer between bilayers. The much higher $d$ value obtained for the interaction of lipid membranes with SNTs loaded with naproxen than for the interaction of lipid membranes with naproxen alone, resides in the fact that the naproxen loaded into the nanotubes is in a higher concentration than the naproxen added alone to the lipid membrane. Therefore, upon the release from the SNTs which is observed at this $\mathrm{pH}$, naproxen is in a higher concentration in the membrane (Fig. 3D-II and Fig. 3B-II). Upon interaction of the loaded SNTs with the lipid membranes at $\mathrm{pH} 5.0$, the phase transition temperature is dislocated to lower values (Fig. 4 (A)). This indicates that naproxen released at this $\mathrm{pH}$ is able to reduce the phase transition temperature of the lipid membrane to $38{ }^{\circ} \mathrm{C}$ in comparison to the $42{ }^{\circ} \mathrm{C}$ obtained in pure DPPC bilayers. Such a significant reduction in the lipid membrane phase transition temperature is consistent with a membrane fluidization effect of naproxen which is required for its therapeutic effect.

\section{Conclusions}

The SNT pH sensitive naproxen delivery system is based on the strength of electrostatic interactions between the drug molecules and the amino groups on the inner surface of the nanotubes, which is mainly determined by the $\mathrm{p} K_{\mathrm{a}}$ value of the drug. At pH 7.4, APTES is completely positively charged, whereas naproxen $\left(\mathrm{p} K_{\mathrm{a}} 4.2\right)$ is almost completely negatively charged, establishing thereby a strong link between the two. At pH 5.0, naproxen becomes more neutral, being only partially ionized. Hence, a much weaker Coulomb interaction is established between naproxen and the protonated amino groups on the interior of the SNTs, contributing considerably to a preferable drug release at $\mathrm{pH}$ 5.0. Indeed, at this $\mathrm{pH}$, the SAXS profile is similar to the one obtained for naproxen:DPPC mixtures, confirming thereby the release of the drug from the inner voids of the nanotubes.

At $\mathrm{pH} 7.4$ the SNTs loaded with naproxen produced a negligible effect in the lipid bilayers, once the drug is protected inside of the nanotubes, it is not released in the membrane, and for this reason the drug is not able to disturb the lipid structure.

Considering the lipid phase, the effect of the nanocarrier system loaded with the drug on the $\mathrm{L}_{\alpha}$ phase of the lipid membrane can be associated with the anti-inflammatory efficiency of this system. Actually, to be efficient, NSAIDs must pass through several cell membranes until they reach their target enzyme (COX) which also has a membrane location. According to this, it is possible to say that the SNTs loaded with naproxen are able to release the drug at the $\mathrm{pH}$ of the inflamed tissues and they are also able to interact with the 
$\mathrm{L}_{\alpha}$ phase of the membrane, which is required in order to exert the anti-inflammatory effects in vivo.

Moreover, as the SNTs alone did not perturb the membrane's organizational properties, it can be concluded that SNT uptake does not involve insertion and diffusion of the nanotubes across cell membranes, as described for carbon nanotubes. ${ }^{18}$

\section{Acknowledgements}

C. N. thanks FCT (Fundação para a Ciência e Tecnologia) for the Post-Doc Grant (SFRH/BPD/81963/2011). M. P. P. and C. T. S. are thankful to FCT for doctoral and post-doctoral Grants SFRH/BD/43440/2008 and SFRH/BPD/82010/2011, respectively. A. Apolinario acknowledges the financial support under project PTDC/EQU-EQU/107990/2008. J. P. A. thanks the Fundação Gulbenkian for its financial support within the "Programa Gulbenkian de Estílmulo à Investigacão Científica." The authors acknowledge funding from FCT through the Associated Laboratory-IN. The authors also thank HASYLAB at DESY, Hamburg, Germany, for beam time and support through the project I-20090160 EC. We are also grateful to Dr Sérgio Funari for all the help at the beamline A2.

\section{References}

1 S. J. Son, X. Bai, A. Nan, H. Ghandehari and S. B. Lee, J. Controlled Release, 2006, 114, 143-152.

2 R. K. Sharma, S. Das and A. Maitra, J. Colloid Interface Sci., 2005, 284, 358-361.

3 M. Shimada, S. Natsugoe, K. Tokuda, T. Kumanohoso, K. Nakamura, K. Yamada and T. Aikou, 23rd International
Symposium on Controlled Release of Bioactive Materials, 1996 Proceedings, 1996, 387-388.

4 C. Barbe, J. Bartlett, L. G. Kong, K. Finnie, H. Q. Lin, M. Larkin, S. Calleja, A. Bush and G. Calleja, Adv. Mater., 2004, 16, 1959-1966.

5 P. K. Jal, S. Patel and B. Mishra, Talanta, 2004, 62, 1005-1028.

6 S. J. Son, X. Bai and S. B. Lee, Drug Discovery Today, 2007, 12, 650-656.

7 S. Ono, M. Saito and H. Asoh, Electrochim. Acta, 2005, 51, 827-833.

8 C. Hawkins and G. W. Hanks, J. Pain Symptom Manage., 2000, 20, 140-151.

9 A. Angelova, B. Angelov, V. M. Garamus, P. Couvreur and S. Lesieur, J. Phys. Chem. Lett., 2012, 3, 445-457.

10 O. Jordan, N. Butoescu and E. Doelker, Eur. J. Pharm. Biopharm., 2009, 73, 205-218.

11 H. Masuda and K. Fukuda, Science, 1995, 268, 1466-1468.

12 D. C. Leitao, A. Apolinario, C. T. Sousa, J. Ventura, J. B. Sousa, M. Vazquez and J. P. Araujo, J. Phys. Chem. C, 2011, 115, 8567-8572.

13 C. T. Sousa, C. Nunes, M. P. Proenca, D. C. Leitao, J. L. F. C. Lima, S. Reis, J. P. Araujo and M. Lucio, Colloids Surf., B, 2012, 94, 288-295.

14 C. Nunes, G. Brezesinski, J. L. F. C. Lima, S. Reis and M. Lucio, J. Phys. Chem. B, 2011, 115, 8024-8032.

15 C. Nunes, G. Brezesinski, J. L. F. C. Lima, S. Reis and M. Lucio, Soft Matter, 2011, 7, 3002-3010.

16 C. Bourgaux, B. Pili, H. Amenitsch, G. Keller, S. LepetreMouelhi, D. Desmaele, P. Couvreur and M. Ollivon, Biochim. Biophys. Acta, Biomembr., 2010, 1798, 1522-1532.

17 R. Winter, in Chemistry at Extreme Conditions, ed. M. R. Manaa, B. V. Elsevier, 1st edn, 2004, pp. 29-82.

18 H. J. Dai, N. W. S. Kam and Z. A. Liu, Angew. Chem., Int. Ed., 2006, 45, 577-581. 